\title{
Investigating the Role of Lipid Transfer in Microbubble-Mediated Drug Delivery
}

\author{
Miles Aron, ${ }^{\dagger}$ Oliver Vince, ${ }^{\dagger}$ Michael Gray, Christophoros Mannaris, and Eleanor Stride*(0) \\ Institute of Biomedical Engineering, University of Oxford, Old Road Campus Research Building, Oxford OX3 7DQ U.K.
}

Supporting Information

ABSTRACT: Sonoporation, the permeabilization of cell membranes following exposure to microbubbles and ultrasound, has considerable potential for therapeutic delivery. To date, engineering of microbubbles for these applications has focused primarily upon optimizing microbubble size and stability, or attachment of targeting species and/or drug molecules. In this work, it is demonstrated that the microbubble coating can also be tailored to directly influence cell permeabilization. Specifically, lipid exchange mechanisms between phospholipid microbubbles and cells can be exploited to significantly increase sonoporation efficiency in vitro. A theoretical analysis of the energy required for pore formation was carried out. From this, it was hypothesized that sonoporation could be promoted by the transfer of lipid molecules with appropriate carbon chain length and/or shape (cylindrical or conical). Spectral imaging with a hydration-sensitive membrane probe (C-Laurdan) was used to measure changes in the membrane lipid order of A-549 cancer cells following exposure to suspensions of different phospholipids. Two candidate lipids were identified, a short-chain-length phospholipid (1,2-dilauroyl-sn-glycero-3-phosphocholine (DLPC)) and a medium-chain-length lysolipid (1-palmitoyl-2hydroxy-sn-glycero-3-phosphocholine (16:0 lyso-PC)). Microbubbles were prepared with matched concentrations, size distributions, and acoustic responses. Confocal microscopy was used to measure cell uptake of a model drug (propidium iodide) with and without ultrasound exposure $(1 \mathrm{MHz}, 250 \mathrm{kPa}$ peak negative pressure, $1 \mathrm{kHz}$ pulse repetition frequency, $10 \%$ duty cycle, 15 s exposure). Despite significantly decreasing the cell membrane lipid order, DLPC did not increase sonoporation. Microbubbles containing 16:0 lyso-PC, however, produced a $\sim 5$-fold increase in sonoporation compared to control microbubbles. Importantly, the lyso-PC molecules were incorporated into the microbubble coating and did not affect cell permeability prior to ultrasound exposure. These findings indicate that microbubbles can be engineered to exploit lipid exchange between microbubble shells and cell membranes to enhance drug delivery, a new optimization route that may lead to enhanced therapeutic efficacy of ultrasound-mediated treatments.

\section{INTRODUCTION}

Exposure to microbubbles (MBs) and ultrasound can induce an increase in cell permeability (sonoporation) offering a promising modality for noninvasively increasing the local uptake of therapeutics in a range of applications, including cancer and neurodegenerative diseases. ${ }^{1-4}$ A range of potential mechanisms have been proposed to explain the process of sonoporation, but this remains an area of active research., Recent studies have reported the shedding of surface material from phospholipid-coated $\mathrm{MBs},{ }^{7}$ the deposition of liposomal lipids onto cell membranes, ${ }^{8}$ and the transfer of lipids from phospholipid-shelled MBs to synthetic membranes during ultrasound excitation in vitro. ${ }^{9}$ Previous work by the authors has also shown that lipid transfer from MBs to cell membranes influences membrane hydration and lipid order. ${ }^{10}$ Critically, this effect was found to be highly dependent on the $\mathrm{MB}$ formulation (i.e., carrier lipid chain length, emulsifier species, and molar ratio of lipid to emulsifier). Only a few studies have evaluated the biodistribution of lipids following ultrasoundmediated drug delivery in vivo, but cavitation-assisted deposition of lipids in the rat kidney has been demonstrated in vivo using microPET and 18F-labeled lipid, and these results suggest that lipid deposition is likely to be occurring in many ultrasound studies. ${ }^{11}$ What is not known, however, is whether this lipid deposition has any influence on sonoporation or other ultrasound/cavitation-mediated bioeffects. Addressing this question was the aim of the present study.

The first objective was to investigate whether the reduction in cell membrane lipid order resulting from lipid transfer from MBs also lowers the energy barrier to pore formation in cell membranes. As pore formation is thought to be a principal pathway for drug uptake in sonoporation, it was also hypothesized that lipids that promote/stabilize membrane pores and/or decrease cell membrane lipid order would increase sonoporation efficiency if transferred into the cell

Received: August 1, 2019

Revised: September 13, 2019

Published: September 13, 2019 
membrane. The theoretical basis for this hypothesis is as follows:

The net energy associated with the formation of a single cylindrical pore of radius $R$ in a lipid membrane (Figure 1 ) is given by $^{12}$

$$
\Delta E=2 \pi R \gamma-\pi R^{2} \Gamma
$$

where $\gamma$ is the line tension (in $\mathrm{N}$ ) and $\Gamma$ is the interfacial tension (in $\mathrm{N} / \mathrm{m}$ ).

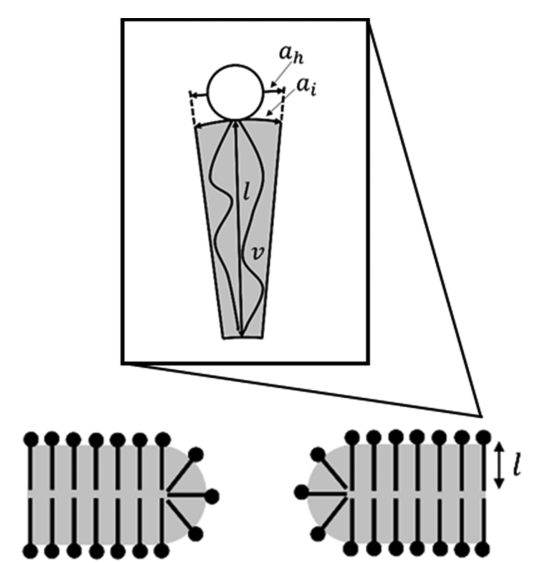

Figure 1. Diagram showing the molecular arrangement and dimensions used to determine the line tension around a pore $\left(\right.$ modified from May ${ }^{13}$ ). Lipid head groups are dots, and lipid chains are simplified to a single line per lipid. It can be seen that the pore size used to determine line tension is equal to the bilayer thickness. Inset: the variables used in determining the line tension are labeled on a diagram of a single lipid $\left(a_{\mathrm{i}}\right.$ is the molecular cross-sectional area at the hydrocarbon-water interface, $a_{\mathrm{h}}$ is the head group area, $l$ is the average chain length, and the chain volume, $v$, is given by $\left.v=l a_{\mathrm{h}}\right)$.

The line tension of a porated membrane is the excess free energy per unit length of the membrane edge when compared to the free energy of an unperturbed lipid bilayer. ${ }^{13}$ Interfacial tension is a measure of the energy per unit area of the cell membrane arising from the imbalance of intermolecular forces between the lipid membrane and surrounding fluid. Therefore, the first term in (eq 1) relates to the energy required to disrupt the membrane; the second term relates to the energy released by a reduction in the surface area of the bilayer. Pores with a radius above a critical value, $R_{\mathcal{c}}$, will grow to rupture the membrane

$$
R_{\mathrm{c}}=\frac{\gamma}{\Gamma}
$$

Substituting eq 2 into eq 1 , it can be seen that the critical energy for pore formation is given by

$$
\Delta E_{c}=\pi \frac{\gamma^{2}}{\Gamma}
$$

While many studies determine the line tension by treating the pore as a deformation in an elastic solid, the assumption of small deformations is violated under this approach. An alternative method for defining the line tension is to consider the attractive and repulsive forces acting on the lipids in the bilayer, as described by Israelachvili et al. ${ }^{14}$ and May. ${ }^{13}$ This model assumes that the pore has a diameter equal to the bilayer thickness (defined as twice the average lipid chain length) and includes only contributions to line tension from the uniform packing of lipids. The molecular free energy of the lipids can be described by

$$
f=\Gamma a_{\mathrm{i}}+\frac{B}{a_{\mathrm{h}}}
$$

where $B$ is the lipid head group repulsion strength, $a_{\mathrm{i}}$ is the molecular cross-sectional area at the hydrocarbon-water interface (e.g., interfacial area), and $a_{\mathrm{h}}$ is the head group area.

It can be seen that at equilibrium, the molecular areas in the bilayer are given by

$$
a_{\mathrm{h}, \mathrm{eq}}=a_{\mathrm{i}, \mathrm{eq}}=\sqrt{\frac{B}{\Gamma}}
$$

Under the assumption that lipid hydrophobic chains are incompressible, the chain volume, $v$, is given by $v=l a_{\mathrm{h}}$, where $l$ is the average chain length. As the lipid head groups spread out to accommodate the presence of the pore, the interfacial area along the pore diameter is increased such that $a_{\mathrm{i}, \mathrm{e}} \sim 2 a_{\mathrm{h}, \mathrm{eq}}$. This can be visualized as the transformation of a cylinder-shaped lipid in the bilayer into a cone-shaped lipid along the pore with chain volume conserved. It follows that the line tension (the excess free energy per unit length of the edge of the pore) is given by

$$
\gamma=\left[f\left(2 a_{\mathrm{h}, \mathrm{eq}}\right)-f\left(a_{\mathrm{h}, \mathrm{eq}}\right)\right] N
$$

where $N=\frac{\pi l^{2}}{2 v}$ is the number of lipids in the pore per unit length (e.g., by dividing the micellar area by the chain volume). This results in a useful expression for the line tension

$$
\gamma=\frac{\pi}{4} \Gamma l
$$

The critical energy required for pore formation is thus

$$
\Delta E_{\mathrm{c}}=\frac{\pi^{3}}{16} \Gamma l^{2}
$$

From eq 5 , the interfacial tension can be expressed as $\Gamma=\frac{B}{a_{\mathrm{h}, \text { eq }}^{2}}$ such that

$$
\Delta E_{\mathrm{c}}=\frac{\pi^{3}}{16} B\left(\frac{l}{a_{\mathrm{h}, \mathrm{eq}}}\right)^{2}
$$

From eq 9, it may be seen that the energy required for pore formation is directly related to the characteristics of the lipid molecules. Following directly from this, the second objective of this study was to determine whether an MB formulation could be deliberately designed to sensitize cells to ultrasoundmediated delivery of a model drug via lipid exchange mechanisms.

\section{MATERIALS AND METHODS}

2.1. Overview of Experiments. Multiple materials have been used for the stabilization of microbubbles, ${ }^{15}$ including cross-linked serum albumin, ${ }^{16,17}$ biopolymers such as polylactic acid (PLA) and polylactic co-glycolic acid (PLGA), ${ }^{4,18,19}$ and biological surfactants, most notably phospholipids. ${ }^{2}$ Of the four commercially available microbubble agents worldwide, three are based on phospholipids combined with lipid emulsifiers (SonoVue ${ }^{(R)}$ Bracco, Definity ${ }^{(R)}$ Bristol Myers Squibb, and Sonazoid ${ }^{(\mathrm{R})}$ GE Healthcare). Thus, the present study has focused on the effect of lipid microbubble composition on sonoporation, although it should be noted that the effects of other coating materials upon cellular membranes are also 
worthy of investigation. From eq 9, it may be seen that both the hydrocarbon chain length and symmetry of the lipid molecules can potentially influence pore formation. These were also found to be important factors in determining material transfer in the authors' previous results, ${ }^{10}$ as was their relative concentration. The following experiments were therefore carried out.

2.1.1. Effect of Excess Emulsifier. Microbubbles consisting of either 1,2-distearoyl-sn-glycero-3-phosphocholine (DSPC) or 1,2-dibehenoyl-sn-glycero-3-phosphocholine (DBPC) and polyoxyethylene (40) stearate (PEG40S) were prepared as described below. These formulations were selected as they are commonly used in microbubble research. $^{20-22}$ The commercial contrast agent SonoVue ${ }^{(\mathrm{R})}$ whose major constituent is DSPC in combination with a PEG derivative was also prepared according to the manufacturer's instructions. Samples of microbubbles with matched concentrations and size distributions were incubated with A-549 cells either as prepared or following "washing" by centrifugation to remove excess emulsifier from the suspending liquid. The effect on the lipid order of the cell membranes was measured using spectral imaging (Section 2.6), and the number of cells permeabilized to a model drug following ultrasound exposure was determined by confocal microscopy (Section 2.8).

2.1.2. Effect of Hydrocarbon Chain Length. Equation 9 indicates that the transfer of lipid molecules with shorter hydrocarbon chain into the cell membrane should reduce the energy required for pore formation by lowering the average lipid chain length. Mismatches in lipid chain lengths in a lipid bilayer that alter local hydration and membrane curvature can also act to destabilize the membrane. A-549 cells were therefore incubated with concentration-matched suspensions of phosphatidylcholines (PCs) of different chain lengths (from 10 to 22), and changes in membrane lipid order were again measured by spectral imaging. Further investigation was then carried out into the effect upon A-549 cell sonoporation of the PC (1,2-dilauroyl-snglycero-3-phosphocholine, DLPC) most likely to nontoxically decrease the lipid order of the cell membrane in the time scales of a practical sonoporation experiment $(<10 \mathrm{~min})$.

2.1.3. Effect of Lipid Molecule Shape. Equation 9 also indicates that as a lipid becomes more conical (either as $a_{\mathrm{h}}$ is increased, $l$ is decreased, or both, for the same chain volume), it will have greater potential to promote and stabilize membrane pores. Indeed, this concept has been employed in the design of thermosensitive liposomes that use conical lyso-PCs to stabilize pores in the liposome upon exposure to mild hyperthermia, enabling triggered drug release. ${ }^{23}$ It was therefore hypothesized that saturated lysophosphatidylcholines (lyso-PCs) might be more effective than short-chain phospholipids in promoting sonoporation. The effects of lyso-PC on cell membrane lipid order and permeability were evaluated as above, and, due to the widespread permeabilizing effects of free lyso-PC, lyso-PC-loaded MBs (lyso-MBs) were then developed for the ultrasound-triggered release of lyso-PC. Lyso-MBs were then assessed for their ability to potentiate sonoporation.

2.2. MB Manufacture. MBs were produced using a previously reported batch sonication protocol. ${ }^{24}$ For the purposes of this study, it was important to be able to produce populations that were consistent in terms of size distribution, concentration, stability, and acoustic response. While sonication has several shortcomings as a fabrication process in terms of the polydispersity of the microbubbles, it does meet the above requirements. Moreover, it is still widely used in the manufacture of commercial agents and in the research literature ${ }^{25,26}$ and thus enables comparison of microbubble characteristics with those reported in other studies. Briefly, the relevant lipids (Avanti Polar Lipids Inc.) and polyoxyethylene (40) stearate (PEG40S, Sigma-Aldrich, U.K.) were dissolved in chloroform (Sigma-Aldrich, U.K.) and mixed in a glass vial at the molar ratios given in Table 1 (total weight of constituents $=20 \mathrm{mg}$ ). The mixture was subsequently heated on a hot plate at $58{ }^{\circ} \mathrm{C}$ for $12 \mathrm{~h}$, to allow for the chloroform to evaporate. This lipid film was suspended in $5 \mathrm{~mL}$ of filtered phosphate-buffered saline (PBS, $\mathrm{pH}$ 7.4) solution (ThermoFisher Scientific Loughborough, U.K.) for $1 \mathrm{~h}$ on a hot plate at $100{ }^{\circ} \mathrm{C}$ under constant magnetic stirring. Lipids were then homogeneously dispersed for $90 \mathrm{~s}$ using a sonicator (XL 2000, probe diameter 3
Table 1. Overview of the MB Formulations Used in This Study

\begin{tabular}{llll} 
& \multicolumn{1}{c}{ formulation } & \multicolumn{1}{c}{$\begin{array}{c}\text { lipids and } \\
\text { emulsifiers }\end{array}$} & \multicolumn{1}{c}{ molar ratio } \\
i & 18:0 PC-PEG40S & DSPC, PEG40S & $9: 1$ \\
ii & $22: 0$ PC-PEG40S & DBPC, PEG40S & $9: 1$ \\
iii & Sonovue ${ }^{(\mathrm{R})}$ & DSPC, & $71: 9806: 75: 46$ \\
& & PEG4000, & \\
& & DPPG, palmitic & \\
& & acid & \\
iv & Lyso-MBs $(25 \mu \mathrm{M}) /(80 \mu \mathrm{M})$ & DBPC, PEG40S, & $9: 1: 1$ or $9: 1: 4$ \\
& & $16: 0$ lyso-PC & \\
v & PC-MBs & DBPC, PEG40S, & $9: 1: 1$ or $9: 1: 4$ \\
& & $16: 0$ PC & \\
& &
\end{tabular}

$\mathrm{mm}, 20 \mathrm{~W}, 22.5 \mathrm{kHz}$, Misonix) with the tip completely immersed in the lipid solution (power setting 4). MBs were then formed by placing the sonicator tip at the air-water interface under a constant sulfur hexafluoride $\left(\mathrm{SF}_{6}\right)$ flow (The BOC Group plc, U.K.) and sonicating for $30 \mathrm{~s}$ (power setting 14). Immediately after production, the vial containing the $\mathrm{MB}$ suspension was capped and placed in ice for at least $10 \mathrm{~min}$. A summary of the different microbubble formulations used in the experiments is shown in Table 1.

2.3. MB Size, Stability, and Concentration Matching. To ensure that the microbubbles used in each experiment were matched in terms of size distribution and concentration, these quantities were measured by optical microscopy for each sample prior to their use. MB suspension of $10 \mu \mathrm{L}$ was transferred onto a Neubauer improved cell counting chamber (Hausser Scientific Company) under a $24 \mathrm{~mm}$ $\times 24 \mathrm{~mm}$ glass coverslip (VWR International). At least 10 images of MBs were acquired at a $40 \times$ magnification using a Leica DM500 microscope (Leica Microsystems GmbH, Germany) coupled with a CCD camera (MicroPublisher 3.3 RTV, QImaging, Canada). MB sizing and counting were performed using a purpose-written code in MATLAB (The MathWorks Inc., Natick MA). ${ }^{2}$

Only phospholipid/emulsifier-coated MBs were included in the present study as these are currently the most commonly used clinically. Previous authors have used electron microscopy to show that MBs of this type consist of a gas core surrounded by a surfactant monolayer. $^{28}$ The shell "thickness" would thus be expected to be proportional to the size of the constituent molecules. Whereas it is possible to image differences in shell thickness for polymer-coated MB with relative thick shells, ${ }^{29}$ to the best of the authors' knowledge, there is no technique that has sufficient spatial resolution to discern any differences in thickness for the MB formulations used here, as these would be of the order of a few angstroms. ${ }^{30}$ Borden and Longo have, however, shown that phospholipid chain length does affect microbubble stability and acoustic response. ${ }^{31}$ These properties were therefore measured to verify that, between those formulations for which a difference in sonoporation was observed, there was no statistically significant difference in acoustic response as a function of time (SI Sections S5 and S6).

2.4. Removal of Unincorporated Emulsifier. MBs were washed by centrifugation. ${ }^{32}$ Briefly, MBs were loaded into a $10 \mathrm{~mL}$ syringe and centrifuged for $5 \mathrm{~min}$ at $200 \mathrm{~g}$. Following centrifugation, the subnatant was discarded and the MBs were resuspended in phosphate-buffered saline (PBS). It was found that roughly half of the MBs were lost to centrifugation upon washing. However, MBs were concentration-matched with unwashed MBs before experimentation, and, as above, it was verified that there was no statistically significant effect upon the microbubble acoustic response (SI Sections S5 and S6).

2.5. Cell Culture. Immortalized human alveolar adenocarcinomic cells (A-549s) were grown in Dulbecco's modified Eagle medium (DMEM) with $10 \%$ fetal bovine serum (FBS) and $1 \%$ penicillin/ streptomycin $(\mathrm{P} / \mathrm{S})$. Cells were grown in a temperature- and $\mathrm{CO}_{2}-$ controlled incubator at $37{ }^{\circ} \mathrm{C}$ and $5 \% \mathrm{CO}_{2}$, respectively. Cells were removed from T-75 culture flasks at an $\sim 80 \%$ confluence by a $\sim 2 \mathrm{~min}$ exposure to $0.25 \%$ trypsin/EDTA. Cells were then suspended in a 10 


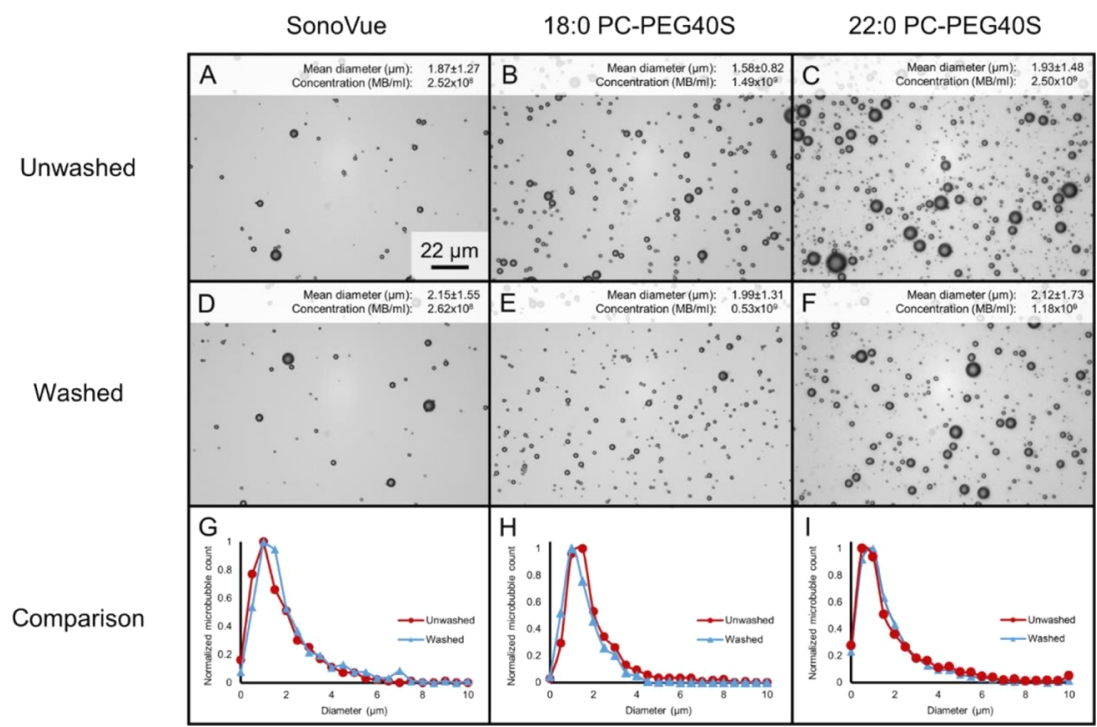

Figure 2. Effect of washing various MB formulations at $22^{\circ} \mathrm{C}$. Unwashed MBs are shown in panels (A-C). Washed MBs are shown in panels (DF). A comparison of the normalized size distributions of washed and unwashed MBs is shown in panels (G-I). The columns from left to right contain SonoVue MB, 18:0 PC-PEG40S MB, and 22:0 PC-PEG40S MB, respectively. Images were taken with a 40× magnification following a 10× dilution. The scale bar in (A) applies to all panels.

$\mathrm{mL}$ culture medium (DMEM with $10 \% \mathrm{FBS}$ ) to deactivate the trypsin and centrifuged for $5 \mathrm{~min}$ at $\sim 200 \mathrm{~g}$ to form a pellet. Cells were then resuspended in a $10 \mathrm{~mL}$ culture medium. Cell concentration and viability were measured using trypan blue and a Countess Automated Cell Counter (Invitrogen). Cells were plated at a $\sim 40 \%$ seeding density in $35 \mathrm{~mm}$ Ibidi $\mu$-Dishes for sonoporation experiments and 8well Ibidi $\mu$-Slides (Ibidi, Gräfelfing, Germany) for spectral imaging experiments and were used in experiments $\sim 16 \mathrm{~h}$ after plating. Subconfluent cells were employed to improve the reliability of image segmentation and to reduce uncertainty introduced by the varying tightness of cell-cell contacts at confluence. All cell culture materials were purchased from ThermoFisher Scientific, unless stated otherwise.

2.6. Measuring Cell Membrane Lipid Order Using Spectral Imaging. Changes in the lateral order of the lipids (revealing how densely the lipids are packed) due to exposure to ultrasound and/or MBs were quantified using C-Laurdan, an environmentally sensitive fluorescent probe with similar spectral characteristics to Laurdan but with greater photostability. C-Laurdan fluorescence emission spectra were measured for each pixel at a $63 \mathrm{X}$ magnification by spectral imaging on a confocal microscope equipped with a 32-channel GaAsP detector array (Zeiss LSM 780, Carl Zeiss AG, Germany), following a method previously reported. ${ }^{33} \mathrm{C}$-Laurdan was excited at $405 \mathrm{~nm}$, and the lambda detection range was set between 415 and $691 \mathrm{~nm}$. Generalized polarization (GP, ranging from -1 to 1 ) was employed as a relative measure of lipid order $^{34}$ and was calculated as follows

$$
G P=\frac{I_{440}-I_{490}}{I_{440}+I_{490}}
$$

where $I_{440}$ and $I_{490}$ correspond to the fluorescence intensity at 440 and $490 \mathrm{~nm}$ emission wavelengths, respectively. Notably, $440 \mathrm{~nm}$ is associated with lower hydration and polarity, resulting in GP $=+1$ when only this wavelength is emitted. This indicates higher lipid order and means that lipids are in the gel phase (ordered). Conversely, 490 $\mathrm{nm}$ is associated with greater hydration and polarity, resulting in GP = -1 . This indicates lower lipid order and prevalence of a liquidcrystalline phase (disordered, or less ordered).

Cells were washed with phosphate-buffered saline (PBS) twice and then exposed to MBs in PBS for $\sim 90 \mathrm{~s}$. The MB solution was then removed, cells were washed again with PBS, and labeled with $400 \mathrm{nM}$ C-Laurdan in PBS for $\sim 1$ min before imaging. Temperature control was achieved using the incubation chamber of the confocal microscope and verified using a thermocouple. PBS was preheated in a water bath and also transferred into the temperature-controlled microscope chamber. Samples were measured with a thermocouple after completing imaging to confirm temperature control to within \pm 1 ${ }^{\circ} \mathrm{C}$. A custom-image-processing routine ${ }^{35}$ for batch-processing spectral images was then used to obtain a GP value for both the cells and cell membranes.

2.7. Ultrasound for Sonoporation Experiments. Ultrasound exposure for the sonoporation experiments was conducted in a water tank system consisting of a $1 \mathrm{MHz}$ focused ultrasound source $(8233$ A101, Imasonic, Voray sur l'Ognon, France), a purpose-built sample holder, and a single element focused transducer used as a passive cavitation detector (Panametrics V320, Olympus NDT, Essex, U.K.). For further information on this setup, please see the SI (Section S3). The acoustic absorber was placed opposite the transducer to attenuate reflections. The sample holder was designed to hold cell culture dishes ( $\mu$-Dishes, Ibidi) with plastic substrates much thinner $(\sim 100 \mu \mathrm{m})$ than the acoustic wavelength in water enclosed with a custom poly(dimethylsiloxane) (PDMS) lid ( 1.2 mm thick). Design and characterization of the acoustical properties of this PDMS "Sonolid" have been described in detail by Carugo et al. ${ }^{36}$

In preparation for sonoporation, cells plated in a $35 \mathrm{~mm} \mu$-dish were fitted with a Sonolid. ${ }^{36}$ The assembly was filled by needle injection through the Sonolid inlet with a solution containing $40 \mu \mathrm{L}$ propidium iodide (PI), $10 \mathrm{~mL}$ Dulbecco's phosphate-buffered saline (PBS), and $1 \mathrm{~mL} \mathrm{MBs,} \mathrm{ensuring} \mathrm{that} \mathrm{there} \mathrm{were} \mathrm{no} \mathrm{entrapped} \mathrm{air}$ bubbles. The Sonolid inlet and outlet were then plugged. The dish was then inverted so that the MBs floated into contact with the cells and placed into the ultrasound system. The cells were then exposed to $15 \mathrm{~s}$ of ultrasound at $1 \mathrm{MHz}$, a $0.25 \mathrm{MPa}$ peak negative pressure, and with a $1 \mathrm{kHz}$ pulse repetition frequency at a $10 \%$ duty cycle. These ultrasound settings were found to reliably produce both sonoporation in around $10 \%$ of the cells and negligible cell death by the time the cells were imaged in each control sample.

2.8. Assessment of Cell Sonoporation. Upon removal from the ultrasound system, the cells were washed with PBS and incubated with calcein-AM for $5 \mathrm{~min}$ before imaging. PI and calcein-AM staining were evaluated using dual-wavelength fluorescence microscopy (Nikon Eclipse Ti, Melville, NY) with multipoint scanning to account for inhomogeneity of the ultrasound field and other area effects that may originate from cavitation events. PI is normally cell-impermeant and thus widely used as a model drug as its uptake indicates membrane permeabilization. ${ }^{37}$ Calcein-AM is cell-permeant and converts to green fluorescent calcein upon uptake by viable cells, 


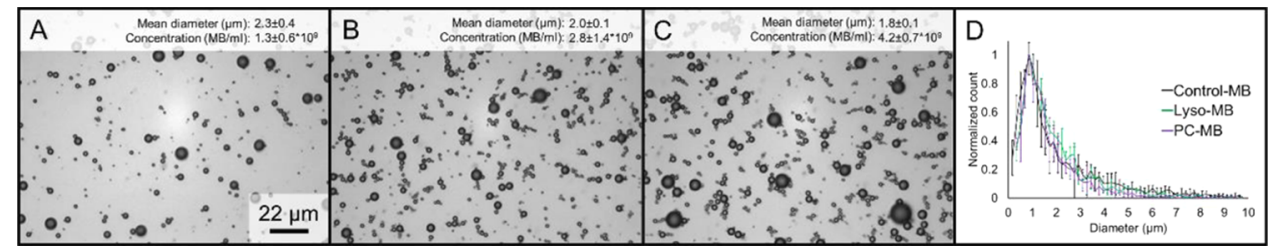

Figure 3. Size distributions and concentrations for MBs used in sonoporation experiments. MB diameters and concentrations for three batches are shown with representative bright-field microscopy images in $(\mathrm{A}-\mathrm{C})$ for control-MB, lyso-MB, and PC-MB formulations.

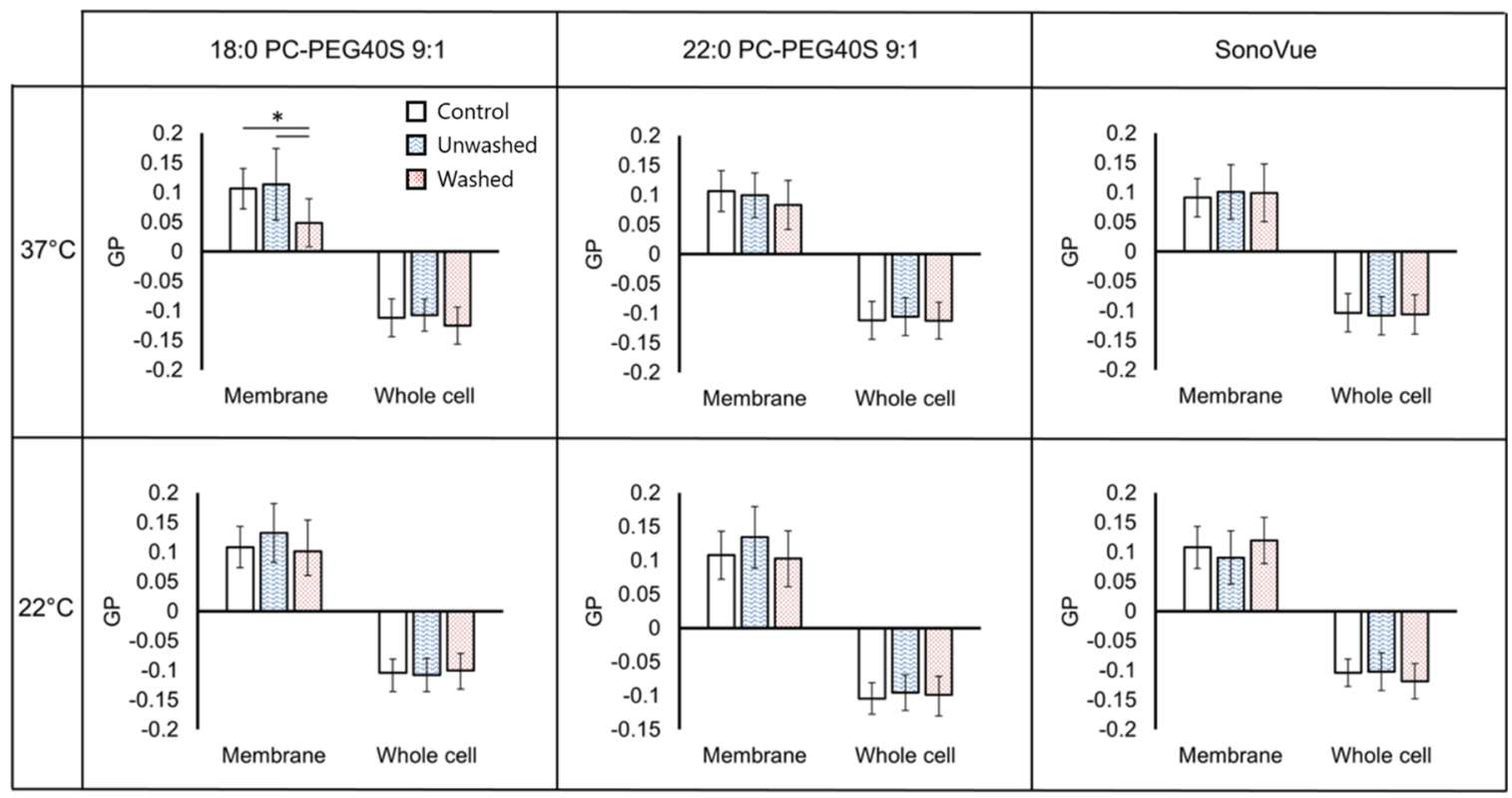

Figure 4. Effect of microbubbles on A-549 cell membrane and intracellular lipid order (GP). Microbubbles tested include (A) 18:0 PC-PEG40S, (B) 22:0 PC-PEG40S, and (C) SonoVue with and without washing by centrifugation at RT and $37{ }^{\circ} \mathrm{C}$, respectively. The only statistically significant result $(p<0.01, n=6)$ is from standard 18:0 PC-PEG40S MB at $37^{\circ} \mathrm{C}$, which reduced cell membrane lipid order.

thus providing a useful indicator of toxicity. ${ }^{38} \mathrm{~A}$ purpose-written MATLAB script was then used to count the number of cells with and without permeabilized membranes in each image. Further details on this script can be found in the SI (Section S4).

2.9. Temperature Control during Sonoporation Experiments. For sonoporation at $37{ }^{\circ} \mathrm{C}$, degassed water was heated in a water bath and poured into the ultrasound system immediately prior to each sonication. A thermocouple was used to verify the temperature of a no-cell sample under equivalent preparation each day of experiments to confirm temperature control to within $\pm 1{ }^{\circ} \mathrm{C}$. For experiments at room temperature (RT), the measured temperature was $22 \pm 1.5^{\circ} \mathrm{C}$.

2.10. Statistical Analysis. A three-way analysis of variance (ANOVA) was performed for experiments with A-549 cells conducted in the $\mu$-Slides using MB formulation, temperature, and washing as factors. A two-way ANOVA with MB formulation and ultrasound exposure as factors was employed for the analysis of the sonoporation results. All ANOVAs with $\alpha$ values below 0.05 were followed with multiple comparisons using Tukey-Kramer tests (significant for $p<0.01$ ). All calculations were performed using Matlab.

\section{RESULTS AND DISCUSSION}

3.1. Microbubble Size Distributions. There were no statistically significant differences between the size distributions of the different formulations shown in Table 1 . These are shown together with representative microscope images in Figures 2 and 3. Figure 2 compares MBs made with a primary phospholipid of a different hydrocarbon chain length (formulations $\mathrm{i}$ and ii) with the commercial contrast agent
SonoVue ${ }^{(\mathrm{R})}$ (formulation iii). It also demonstrates that the washing process had no significant impact on the size distribution.

Lyso-MBs were formed with 22:0 PC, PEG40S, and 16:0 lyso-PC at a 9:1:4 molar ratio such that the lyso-MB solution contained $\sim 315 \mu \mathrm{M}$ of 16:0 lyso-PC (formulation iv). PC-MBs were also produced as a control for the incorporation of a third constituent in the lyso-MBs (formulation v). Figure 3 demonstrates that the incorporation of either 16:0 PC or 16:0 lyso-PC had no effect on the size distribution as compared with that of the control microbubble (formulation ii).

3.2. Effect of Unincorporated Emulsifier on Cell Membrane Lipid Order and Sonoporation. Immortalized human alveolar adenocarcinomic cells (A-549s) stained with C-Laurdan were exposed to different MB formulations (Table 1) made from saturated phosphatidylcholines (PCs) of varying chain lengths and an emulsifier, polyoxyethylene (40) stearate (PEG40s). Changes in the lipid order of the cell membranes were determined by measuring the fluorescence emissions from C-Laurdan before and after MB exposure using spectral imaging and calculating the generalized polarization (GP). The roles of temperature $\left(22\right.$ and $37^{\circ} \mathrm{C}$ ) and $\mathrm{MB}$ washing (removal of unincorporated emulsifier) upon cell lipid order were also investigated. In agreement with the previous study, ${ }^{10}$ the only formulation found to significantly affect the cell membrane lipid order in the absence of ultrasound comprised unwashed 18:0 PC-PEG40S MBs $(n=6, \Delta \mathrm{GP} \sim 0.05)$ (Figure 


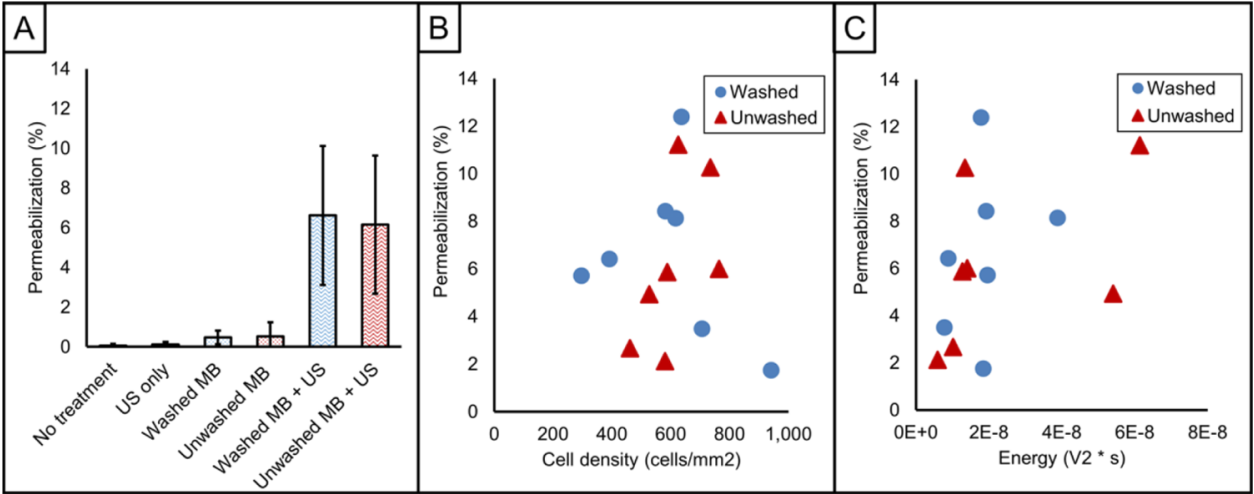

Figure 5. Effect of washing on an 18:0 PC-PEG40S permeabilization efficiency. Permeabilizations for controls (no treatment, ultrasound (ultrasound) only, and washed or unwashed MBs only), as well as treatment conditions (washed or unwashed MBs exposed to ultrasound), are shown in (A). (B) and (C) demonstrate no correlation between either the cell density or the harmonic acoustic emissions and the resultant permeabilization. In (B) and (C), each point is an independent sample. Blue circles represent samples sonoporated with washed MBs, and red triangles represent samples sonoporated with unwashed MBs. Controls are $n=3$. MB + ultrasound conditions are $n=7$.

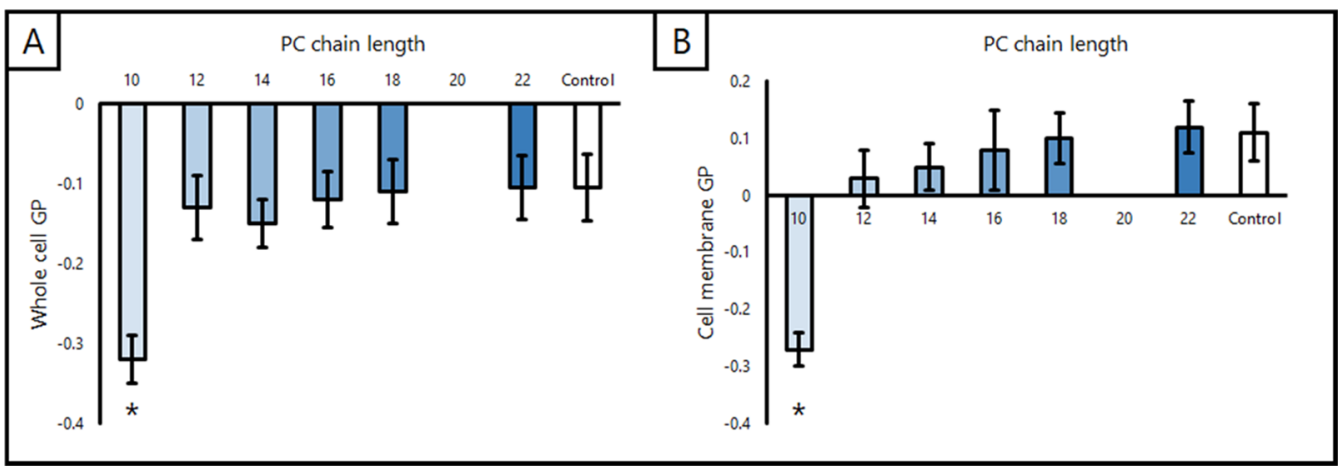

Figure 6. Effect of saturated phosphatidylcholine (PC) lipids of varying hydrocarbon chain lengths of A-549 cell lipid order. Cells were exposed to $5 \mathrm{mM}$ PC at $37{ }^{\circ} \mathrm{C}$ for $\sim 90$ s prior to spectral imaging with C-Laurdan ( $n=5$ each). (A) GP of whole cells and (B) GP of segmented cell membranes.

4). This effect was observed to be temperature-dependent, occurring at $37{ }^{\circ} \mathrm{C}$ but not at $22{ }^{\circ} \mathrm{C}$. As previously found, the cell lipid disordering effect was inhibited by the removal of free lipid and emulsifier from the $\mathrm{MB}$ solution by washing. Interestingly, neither SonoVue nor 22:0 PC-PEG40S MBs had a significant effect on cell membrane lipid order at either 22 or $37{ }^{\circ} \mathrm{C}$, with or without washing. The effect on sonoporation was then investigated by examining the uptake of the dye propidium iodide (PI), to which cells are normally impermeable. However, no difference was observed in cell sonoporation between washed and unwashed 18:0 PCPEG40S MBs $(n=7)$ (Figure 5).

The $\frac{l}{a_{\mathrm{h}, \mathrm{eq}}}$ term in eq 9 can be considered as an analogue to the lipid order, which describes the flexibility of the lipid chains, conventionally by the average angle of the $\mathrm{C}-\mathrm{H}$ bonds with respect to the bilayer normal. For disordered lipids, the chains occupy a larger area and $a_{\mathrm{h}}$ increases while $l$ decreases, resulting in a decrease in the energy required for pore formation. The opposite is true for ordered lipids. Indeed, the permeability of pure lipid bilayers to water molecules, ions, and other small molecules is inversely correlated with lipid order. $^{39,40}$ As exposure to unwashed 18:0 PC-PEG40S MBs has previously been observed to lower cell lipid order, ${ }^{10}$ this exposure was expected to decrease the energy required for pore formation and thus enhance sonoporation. The fact that unwashed 18:0 PC-PEG40S MBs were found to significantly affect the cell lipid order but not sonoporation suggests that the decreased cell membrane lipid order produced by PEG40S alone was insufficient to enhance model drug uptake.

3.3. Effect of PC Chain Length on Cell Lipid Order. A549 cells were also exposed to suspensions of different PCs for $\sim 90 \mathrm{~s}$ at a concentration of $5 \mathrm{mM}$. This concentration and duration of treatment were chosen as it could be effectively translated to the sonoporation setup used. While PCs with a chain length below 16 were expected to affect the thermal behavior of cell lipids, ${ }^{41,42}$ only 10:0 PC (DDPC) was found to induce a significant effect $(p<0.05)$ on the cell membrane and intracellular lipid order after $90 \mathrm{~s}$ exposure (Figure 6). 10:0 PC lowered cell membrane GP by nearly the entire physiological range $^{34}(\Delta \mathrm{GP} \sim-0.38)$, especially compared to that achieved by 18:0 PC-PEG40S MBs described earlier in this study ( $\Delta \mathrm{GP}$ $\sim-0.05)$. Furthermore, the change in membrane GP induced by 18:0 PC-PEG40S MBs was limited to cell membranes, whereas 10:0 PC was also found to disorder intracellular lipids. Further investigation of the effect of 10:0 PC on cell membranes and cytotoxicity is described in the SI (Section S7).

Unlike longer-chain PCs, only very small quantities of shortto medium-chain-length (e.g., 10:0-14:0) PCs are found in biological membranes. These shorter-chain PCs are known to transfer more readily to cell membranes than their longerchain-length counterparts. ${ }^{43}$ Longer exposure times to 12:0 PC did result in statistically significant changes in cell membrane 


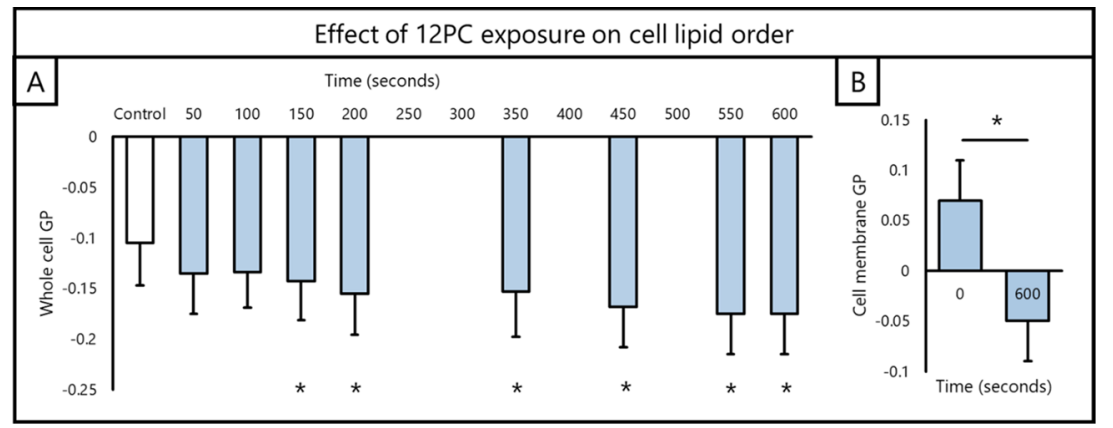

Figure 7. Effect of 12:0 PC on A-549 lipid order. A-549 cells were exposed to $5 \mathrm{mM}$ of $12: 0 \mathrm{PC}$ at $37{ }^{\circ} \mathrm{C}$ for 10 min and measured either concurrently (A) or at the end of the treatment after washing (B), using C-Laurdan and spectral imaging. (A) Significant $(p<0.05)$ decrease in whole-cell lipid order can be observed from a $150 \mathrm{~s}$ exposure onwards. (B) At a 10 min exposure with poststaining, cell membrane GP is also significantly decreased.
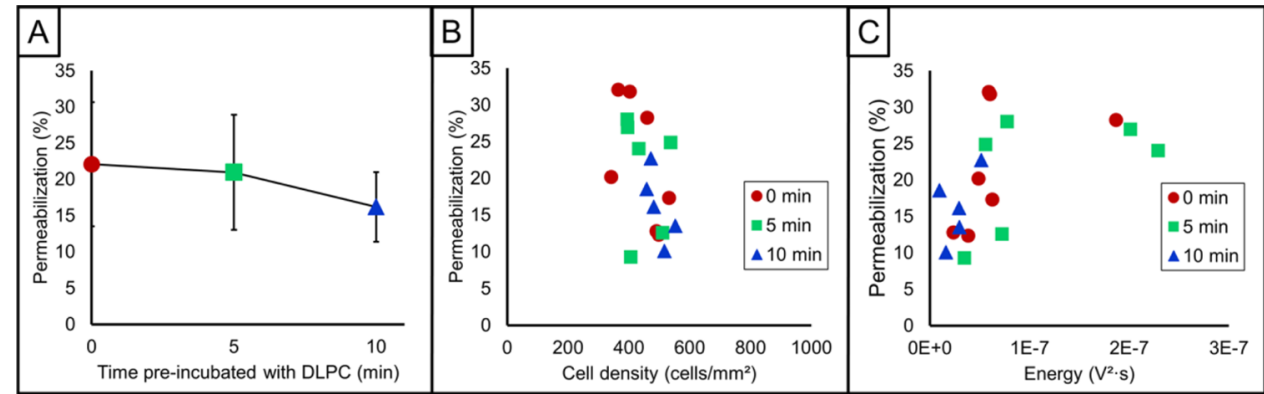

Figure 8. Effect of a 12:0 PC pretreatment on sonoporation efficiency. (A) Mean and standard deviation of permeabilization fraction as a function of a 12:0 pretreatment time $(n=6)$ (also shown in the main paper). (B) Scatter of the permeabilization data vs the observed cell density indicating no dependence between the two variables across the experiments. (C) Scatter of permeabilization data vs the measured harmonic energy in acoustic emissions indicating no dependence between the two variables across the experiments. In (B) and (C), each point represents an independent sonoporation experiment sample for pre-exposure times to 12:0 PC of 0 min (red circles, e.g., no pretreatment), 5 min (green squares), and $10 \mathrm{~min}$ (blue triangles).
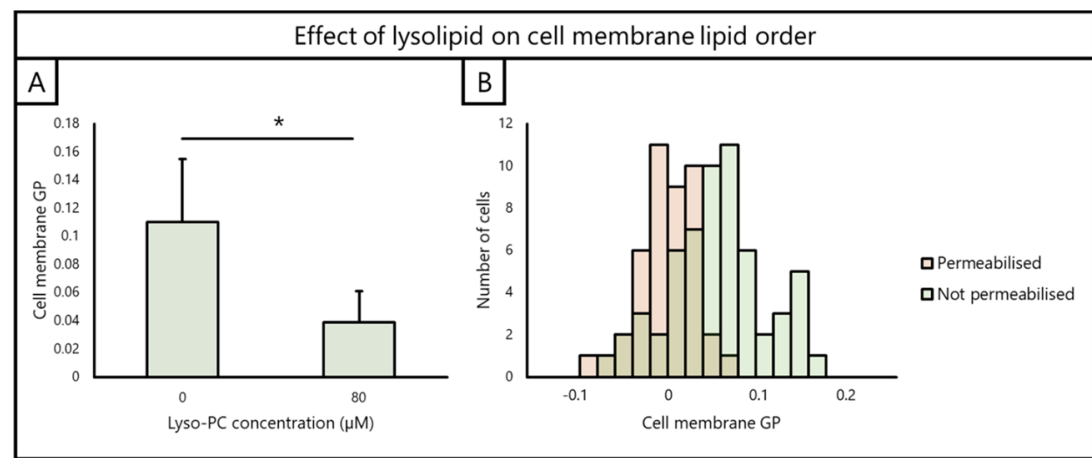

Figure 9. (A) Effect of $16: 0$ lyso-PC at $80 \mu \mathrm{M}$ and $37{ }^{\circ} \mathrm{C}$ on A-549 cell lipid order. Cells were exposed for $\sim 90 \mathrm{~s}(n=3)$. (B) Histograms for cell membrane GP from a sample counterstained with propidium iodide for marking permeabilization after exposure to lyso-PC (without ultrasound) and spectral imaging with C-Laurdan. Cells permeabilized by lyso-PC exposure had lower GP on average $\left(\mathrm{GP}_{\text {permeabilized }}=0.002 \pm 0.034\right.$ and $\left.\mathrm{GP}_{\text {not-permeabilized }}=0.058 \pm 0.055\right)$.

lipid order (Figure 7). Owing to the greater similarity between 12:0 PC and endogenous lipids compared to that of 10:0 PC, it was posited that this lipid would be capable of altering cell lipid order with less severe consequences. It was found that 12:0 PC at $5 \mathrm{mM}$ exhibited a time-dependent lipid disordering effect on A-549 cell membranes and intracellular lipids (Figure 7A). The GP relative to controls is plotted instead of absolute GP to account for drift from repeated exposures of the dye. Using this method, it was found that a $\sim 2$ min exposure to $12: 0$ PC caused a significant disordering of A-549 cell lipids. This effect increased with exposure time until the end of the experiments
( $\sim 10 \mathrm{~min})$. This was confirmed by a more conventional GP measurement (Figure 7B), performed on separate samples exposed to 12:0 PC for $10 \mathrm{~min}$ and then washed and labeled for spectral imaging. Exposure to $5 \mathrm{mM}$ of 12:0 PC for $10 \mathrm{~min}$ lowered cell membrane GP by 0.12 compared to that of control samples on average. This was more than double the change in GP induced by 18:0 PC-PEG40S MBs $(\Delta \mathrm{GP} \sim$ $0.05)$.

3.4. Effect of PCs on Cell Sonoporation. Of the cylindrical PCs investigated, 12:0 PC demonstrated the greatest potential for use in sonoporation experiments. 12:0 


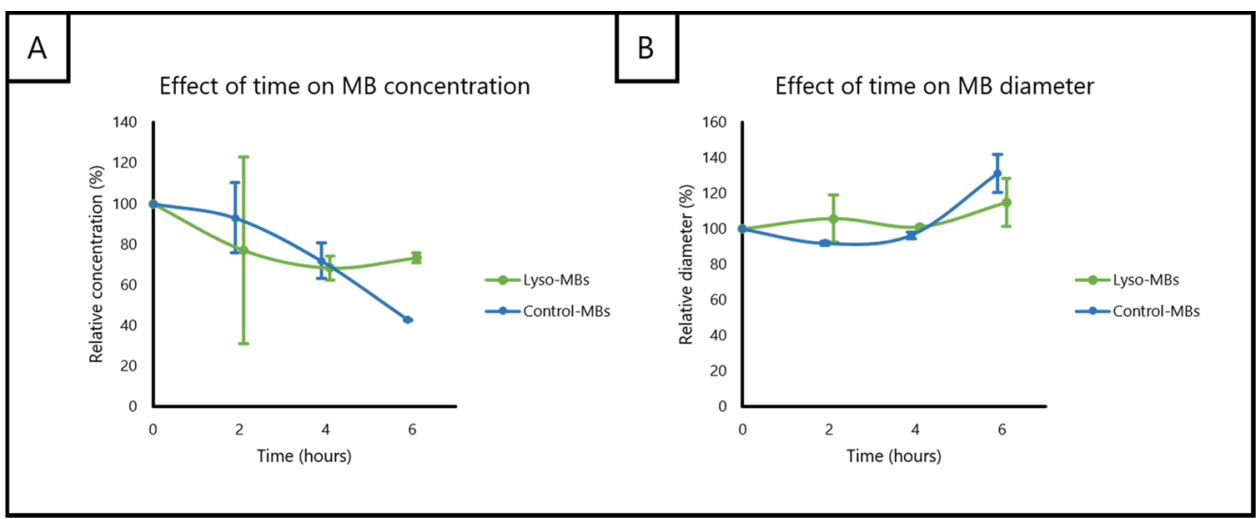

Figure 10. Stability of control-MBs and lyso-MBs as indicated by their diameter and concentration changes over time normalized by their initial values $\left(n=3\right.$; MBs were stored in a sealed vial at $4{ }^{\circ} \mathrm{C}$ in between measurements).

PC was found to disorder cell membrane lipids and intracellular lipids substantially $(\Delta \mathrm{GP} \sim-0.12)$ without the permeabilizing or cytotoxic effects of 10:0 PC. A-549 cells were therefore pretreated with 12:0 PC for either 5 or 10 min before exposure to ultrasound. Pretreatment was used instead of coinjection with $\mathrm{MBs}$ to avoid the added complexity of 12:0 PC potentially altering the acoustic behavior of the MBs. MBs, however, were observed to be stable in the presence of 12:0 PC for the duration of the experiment. Despite the significant effect on cell lipid order, it was found that pretreatment with 12:0 PC had no significant effect on sonoporation (Figure $8 ; p$ $>0.05, n=6$ ).

3.5. Effect of Lyso-PC on Cell Lipid Order. The theoretical analysis above indicated that a different class of lipids, saturated lysophosphatidylcholines (lyso-PCs), might have a more significant effect upon pore formation and stabilization than saturated PCs at the same chain length. Further experiments were therefore carried out to test this hypothesis. 16:0 lyso-PC was found to have a significant disordering effect on cell membrane lipids with $\Delta \mathrm{GP}=-0.058$ \pm 0.038 (Figure 9A). A positive correlation between the uptake of propidium iodide and reduced membrane lipid order was also found (Figure 9B), even without ultrasound exposure. The molarity of 16:0 lyso-PC required for cell membrane permeabilization was found to be 2 orders of magnitude lower than that of 10:0 PC.

3.6. Effect of Lyso-MBs on Cell Sonoporation. After the effects of 16:0 lyso-PC on cell membrane lipid order and permeability had been evaluated, lyso-PC-loaded MBs (lysoMBs) were developed to enable the ultrasound-triggered release of lyso-PC. Figure 10 shows how the concentration and diameter of both control-MBs (formulation ii) and lyso-MBs (formulation iv) changed over time relative to their initial values. As would be expected, the concentration of both formulations decreased over time and the diameter increased. All experiments were conducted within $4 \mathrm{~h}$ of microbubble manufacture when there were no statistically significant differences in stability. Consistent with this finding, lyso-MBs and control-MBs produced statistically indistinguishable acoustic emissions, indicating that their response to ultrasound was very similar (see SI Section S5).

The lyso-MBs were then assessed for their ability to potentiate sonoporation. Lyso-MBs with a final lyso-PC concentration of $80 \mu \mathrm{M}$ were found to significantly increase sonoporation efficiency $(p<0.0001)$, achieving a permeabilization of $39.1 \pm 12.7 \%$ of cells $(n=7)$, compared to $6.0 \pm$
$5.2 \%$ of cells permeabilized following exposure to control-MBs (22:0 PC-PEG40S MBs) and ultrasound $(n=7)$ (Figure 11).

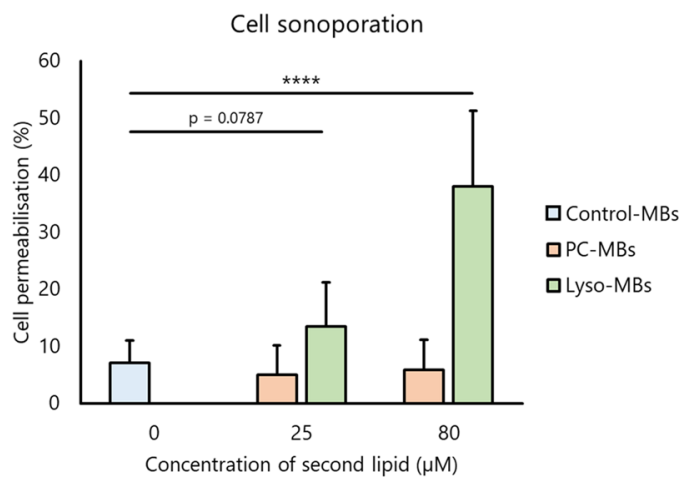

Figure 11. Effect of lyso-MBs and PC-MBs on A-459 cell sonoporation efficiency. Cell permeabilization results are shown for concentrations of 25 and $80 \mu \mathrm{M}$ of 16PC and 16-lyso-PC in the PC$\mathrm{MBs}$ and lyso-MBs, respectively. Lyso-MBs with $80 \mu \mathrm{M}$ of 16 -lyso-PC significantly enhance the delivery of a model drug (propidium iodide) when compared to control-MBs containing lysolipid $(p<0.0001)$. Neither of the PC-MB results are significantly different from that of the control-MBs.

Interestingly, neither 25 nor $80 \mu \mathrm{M}$ lyso-MBs without ultrasound significantly increased the percentage of cells permeabilized when compared to control-MBs without ultrasound (Figure 12). This, along with the effect of $80 \mu \mathrm{M}$ lyso-PC, suggests that lyso-PC was successfully incorporated into the MB shell rather than forming lyso-PC micelles exclusively. If 16:0 lyso-PC had not been incorporated into lyso-MB shells, significant permeabilization would be expected without ultrasound exposure, as observed in Figure 12.

Separately, it was found that adding 16:0 PC lipids to MBs, i.e., to form PC-MBs (as a control for adding lipids and hydrolysis), did not have a significant impact on sonoporation compared to that of control-MBs, despite the fact that some of the $80 \mu \mathrm{M}$ PC-MB samples produced stronger acoustic emissions (see SI Section S6). That PC-MBs did not achieve greater sonoporation provides evidence for attributing the enhancement of sonoporation to the presence of 16:0 lyso-PC in the lyso-MB formulation and not the addition of a third component to the MBs. No dependence of sonoporation on cell density or acoustic emissions was found. 


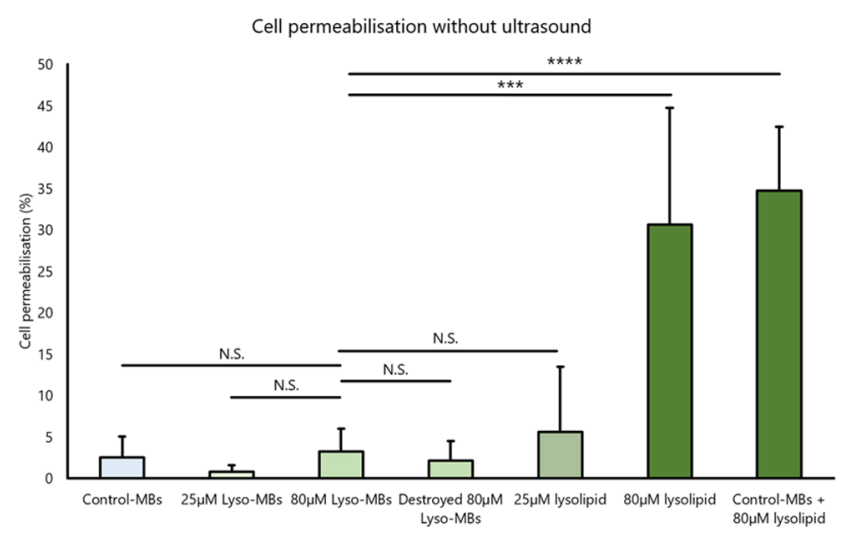

Figure 12. Cell membrane permeabilization MBs, destroyed MBs, and free lysolipid in the absence of ultrasound. Exposure to lyso-MBs, lyso- MBs destroyed by repeated heating to $85{ }^{\circ} \mathrm{C}$ and sonication, or lysolipid at $25 \mu \mathrm{M}$ caused cell permeabilization that was not significantly different from that caused by control-MBs. Exposure to both $80 \mu \mathrm{M}$ of 16:0 lyso-PC and control-MBs spiked with 16:0 lysoPC, however, significantly permeabilized cells $(p<0.0001)$. 16:0 lysoPC was incubated for $90 \mathrm{~s}$, and cells were kept at $37{ }^{\circ} \mathrm{C}$ for all conditions.

To further investigate the effect of the lyso-MBs on cell membrane permeabilization, cells were exposed to either lysoMBs, lyso-MBs destroyed by heating and exposure to lowfrequency ultrasound $(40 \mathrm{kHz})$, or control-MBs with lyso-PC added to the suspending medium immediately prior to exposure for $\sim 90 \mathrm{~s}$ at $37{ }^{\circ} \mathrm{C}$ (see Figure 12). Interestingly, there was no difference in cell permeabilization between cells exposed to lyso-MBs and destroyed lyso-MB. These results again provide evidence that lyso-PC was successfully incorporated into lyso-MB shells rather than forming lyso-PC micelles. In addition, the lyso-MB formulation itself does not appear to be toxic for the exposure conditions tested.

3.7. Potential of Lyso-MBs for Enhanced UltrasoundMediated Therapy. In terms of the expected behavior of lyso-MBs in vivo, the high lipid transfer rate of lyso-PCs and the presence of specific enzymes for its clearance could be beneficial. These factors may enable the rapid delivery of therapeutic material followed by the enzymatic degradation of lyso-PCs into innocuous chemical species. Conversely, there is some risk of toxicity if lyso-PCs transfer from MB shells to blood cells or the endothelium prior to ultrasound exposure in vivo. While this must be evaluated experimentally, it is encouraging that thermosensitive liposomes containing lysoPCs have already been used in clinical trials ${ }^{44}$ and, to the best of the authors' knowledge, no evidence of blood toxicity has been reported.

The 16:0 lyso-PC used in the present study can also likely be replaced by a number of structurally similar synthetic lysolipids (alkyl ether lipids), which may provide additional therapeutic benefit. For example, Edelfosine, similar to lyso-PC but with better metabolic stability in vivo provided by an ether bond, has been shown to inhibit tumor cell proliferation. ${ }^{45}$ Edelfosine was also the first cancer drug to specifically target the cell membrane, with a mechanism involving the disruption of lipid rafts, which ultimately induces apoptosis. ${ }^{46}$ Similarly, Mitelfosine, which is lyso-PC lacking the glycerol residue, has also been investigated to this end, but its clinical translation, like Edelfosine, was hindered due to systemic toxicity. ${ }^{45}$ Perifosine has since been developed in which the choline head group on the Mitelfosine lipid is substituted for a moiety that improves the stability and half-life of the drug. Perifosine has had clinical success when orally administered in combination with other cancer therapies and exhibits selective cancer kill in vitro. ${ }^{45}$ The clinical potential of alkyl ether lipids, either those mentioned or less hemolytic alkyl ether lipids under investigation, potentially could be enhanced when incorporated into an ultrasound-mediated triggered release delivery system.

The microbubble fabrication process could also be improved. As stated above, sonication has a number of limitations, in particular, the relatively broad size distribution of the microbubbles produced and inefficient and/or nonuniform incorporation of coating components. Alternative methods include electrolytic, ${ }^{47}$ electrohydrodynamic, ${ }^{48}$ laserbased, ${ }^{49}$ gyratory, ${ }^{50}$ microfluidic, ${ }^{51}$ and hybrid ${ }^{52}$ approaches, although these still suffer from relatively low production rates. Unnikrishnan et al. ${ }^{53}$ have also recently shown that amalgamation may provide a more efficient process for incorporating targeting species into microbubble coatings, and this might offer a superior method for producing lysoMBs.

\section{CONCLUSIONS}

The aim of this study was to investigate whether the previously reported transfer of material from lipid-coated microbubbles into cell membranes would affect their sensitivity to sonoporation, i.e., permeabilization by ultrasound. The effects of various commonly used microbubble constituents were investigated, specifically the emulsifier polyoxyethylene (40) stearate and phosphatidylcholines (PCs) with varying hydrocarbon chain lengths. The effects of temperature and washing by centrifugation to remove unincorporated lipid and emulsifier were also investigated.

Changes in A-549 cancer cell membrane lipid order following exposure to ultrasound were quantified in terms of changes in generalized potential (GP) using spectral imaging. Permeabilization was measured in terms of the number of cells showing the uptake of a model drug (propidium iodide) before and after ultrasound exposure $(1 \mathrm{MHz}, 250 \mathrm{kPa}$ peak negative pressure, $1 \mathrm{kHz}$ pulse repetition frequency, 10\% duty cycle, 15 $s$ exposure). Of the previously reported microbubble formulations tested, the only one found to significantly affect the cell membrane lipid order in the absence of ultrasound comprised unwashed 18:0 PC-PEG40S MBs $(\Delta \mathrm{GP} \sim 0.05)$ at $37{ }^{\circ} \mathrm{C}$. Upon exposing this formulation, with or without washing, to ultrasound, however, no difference was found in the number of sonoporated cells. Exposing cells to $5 \mathrm{mM}$ suspensions of shorter-chain PC molecules also lowered the membrane lipid order compared to control samples. The suspension of 10:0 PC produced a reduction in GP of 0.38 after $90 \mathrm{~s}$ but was found to be toxic to the cells. The suspension of 12:0 PC was nontoxic and produced a change in GP of 0.12 after $10 \mathrm{~min}$. This was more than double the change in GP induced by 18:0 PC-PEG40S MB, but again, no effect upon sonoporation was observed.

A different class of lipids, saturated lysophosphatidylcholines (lyso-PCs), was also investigated that was hypothesized to transfer more readily into the cell membrane and to promote both the formation and stability of cell membrane pores, as compared with PCs. Suspensions of 16:0 lyso-PC were found to produce a significant reduction in GP (0.058). Although this change was comparable to that produced by 18:0 PC-PEG40S 
microbubbles, cell permeability was seen to increase even in the absence of ultrasound. Microbubbles containing 16:0 lysoPC were therefore prepared and found to produce a $\sim 5$-fold increase in the number of cells permeabilized to a model drug compared to control microbubbles containing 16:0 PC, achieving permeabilization of $39.1 \pm 12.7 \%$ of cells, compared to that of $6.0 \pm 5.2 \%(n=7)$. Importantly, the lyso-PC was found to be incorporated within the microbubble shell and only produced a permeabilizing effect upon exposure to ultrasound. These findings indicate that potential improvements in therapeutic efficacy may be achieved by optimizing the lipid composition of microbubbles to facilitate delivery.

\section{ASSOCIATED CONTENT}

\section{S Supporting Information}

The Supporting Information is available free of charge on the ACS Publications website at DOI: 10.1021/acs.langmuir.9b02404.

Effect of temperature on microbubble size distribution and concentration; lyso-MB size and concentration; ultrasound setup for sonoporation experiments; assessment of cell sonoporation; acoustic characterization and $\mathrm{MB}$ contrast imaging; acoustic emissions during sonoporation experiments; the effect of 10:0 PC on cell lipid order (PDF)

\section{AUTHOR INFORMATION}

\section{Corresponding Author}

*Tel: +44-(0)1865-617 747. Fax: +44-(0)1865-617 728. Email: eleanor.stride@eng.ox.ac.uk.

\section{ORCID $\odot$}

Eleanor Stride: 0000-0003-3371-5929

\section{Author Contributions}

${ }^{\dagger}$ M.A. and O.V. contributed equally to this work.

\section{Notes}

The authors declare no competing financial interest.

The experimental data are available through the University of Oxford ORA data repository (https://ora.ox.ac.uk).

\section{ACKNOWLEDGMENTS}

The authors are grateful to the EPSRC for supporting this research through Grant No. EP/I021795/1 and Programme Grant No. EP/L024012/1.

\section{REFERENCES}

(1) Stride, E. Physical Principles of Microbubbles for Ultrasound Imaging and Therapy. Cerebrovasc. Dis. 2009, 27, 1-13.

(2) Unger, E. C.; Porter, T.; Culp, W.; Labell, R.; Matsunaga, T.; Zutshi, R. Therapeutic Applications of Lipid-Coated Microbubbles. Adv. Drug Delivery Rev. 2004, 56, 1291-1314.

(3) Frenkel, P. A.; Chen, S.; Thai, T.; Shohet, R. V.; Grayburn, P. A. DNA-Loaded Albumin Microbubbles Enhance Ultrasound-Mediated Transfection in Vitro. Ultrasound Med. Biol. 2002, 28, 817-822.

(4) Lentacker, I.; De Geest, B. G.; Vandenbroucke, R. E.; Peeters, L.; Demeester, J.; De Smedt, S. C.; Sanders, N. N. UltrasoundResponsive Polymer-Coated Microbubbles That Bind and Protect DNA. Langmuir 2006, 22, 7273-7278.

(5) Lentacker, I.; De Cock, I.; Deckers, R.; De Smedt, S. C.; Moonen, C. T. W. Understanding Ultrasound Induced Sonoporation: Definitions and Underlying Mechanisms. Adv. Drug Delivery Rev. 2014, 49-64.
(6) Helfield, B.; Chen, X.; Watkins, S. C.; Villanueva, F. S. Biophysical Insight into Mechanisms of Sonoporation. Proc. Natl. Acad. Sci. U.S.A. 2016, 113, 9983-9988.

(7) Luan, Y.; Lajoinie, G.; Gelderblom, E.; Skachkov, I.; van der Steen, A. F. W.; Vos, H. J.; Versluis, M.; De Jong, N. Lipid Shedding from Single Oscillating Microbubbles. Ultrasound Med. Biol. 2014, 40, 1834-1846.

(8) De Cock, I.; Lajoinie, G.; Versluis, M.; De Smedt, S. C.; Lentacker, I. Sonoprinting and the Importance of Microbubble Loading for the Ultrasound Mediated Cellular Delivery of Nanoparticles. Biomaterials 2016, 83, 294-307.

(9) Loughian, C. D.; Seya, P. M.; Pirat, C.; Inserra, C.; Bera, J. C.; Rieu, J. P. Jumping Acoustic Bubbles on Lipid Bilayers. Soft Matter 2015, 11, 3460-3469.

(10) Carugo, D.; Aron, M.; Sezgin, E.; Bernardino de la Serna, J.; Kuimova, M. K.; Eggeling, C.; Stride, E. Modulation of the Molecular Arrangement in Artificial and Biological Membranes by PhospholipidShelled Microbubbles. Biomaterials 2017, 113, 105-117.

(11) Tartis, M. S.; Kruse, D. E.; Zheng, H.; Zhang, H.; Kheirolomoom, A.; Marik, J.; Ferrara, K. W. Dynamic MicroPET Imaging of Ultrasound Contrast Agents and Lipid Delivery. J. Controlled Release 2008, 131, 160-166.

(12) Litster, J. D. Stability of Lipid Bilayers and Red Blood Cell Membranes. Phys. Lett. A 1975, 53, 193-194.

(13) May, S. A Molecular Model for the Line Tension of Lipid Membranes. Eur. Phys. J. E 2000, 3, 37-44.

(14) Israelachvili, J. N.; Marcelja, S.; Horn, R. G. Physical Principles of Membrane Organization. Q. Rev. Biophys. 1980, 13, 121-200.

(15) Sirsi, S. R.; Borden, M. A. Microbubble Compositions, Properties and Biomedical Applications. Bubble Sci., Eng., Technol. 2009, 1, 3-17.

(16) Grinstaff, M. W.; Suslick, K. S. Air-Filled Proteinaceous Microbubbles: Synthesis of an Echo-Contrast Agent. Proc. Natl. Acad. Sci. U.S.A. 2006, 7708-7710.

(17) Mulvana, H.; Browning, R. J.; Tang, M. X.; Hajnal, J. V.; Eckersley, R. J. Albumin Coated Microbubble Optimization: Custom Fabrication and Comprehensive Characterization. Ultrasound Med. Biol. 2012, 1599-1607.

(18) Cui, W.; Bei, J.; Wang, S.; Zhi, G.; Zhao, Y.; Zhou, X.; Zhang, $\mathrm{H}$; $\mathrm{Xu}, \mathrm{Y}$. Preparation and Evaluation of Poly(L-Lactide-CoGlycolide) (PLGA) Microbubbles as a Contrast Agent for Myocardial Contrast Echocardiography. J. Biomed. Mater. Res., Part B 2005, 73, $171-178$.

(19) McEwan, C.; Fowley, C.; Nomikou, N.; McCaughan, B.; McHale, A. P.; Callan, J. F. Polymeric Microbubbles as Delivery Vehicles for Sensitizers in Sonodynamic Therapy. Langmuir 2014, 14926-14930.

(20) Borden, M. A.; Kruse, D. E.; Caskey, C. F.; Zhao, S.; Dayton, P. A.; Ferrara, K. W. Influence of Lipid Shell Physicochemical Properties on Ultrasound-Induced Microbubble Destruction. IEEE Trans. Ultrason. Ferroelectr. Freq. Control 2005, 1992-2002.

(21) van Rooij, T.; Luan, Y.; Renaud, G.; van der Steen, A. F. W.; Versluis, M.; de Jong, N.; Kooiman, K. Non-Linear Response and Viscoelastic Properties of Lipid-Coated Microbubbles: DSPC versus DPPC. Ultrasound Med. Biol. 2015, 41, 1432-1445.

(22) Wu, S. Y.; Chen, C. C.; Tung, Y. S.; Olumolade, O. O.; Konofagou, E. E. Effects of the Microbubble Shell Physicochemical Properties on Ultrasound-Mediated Drug Delivery to the Brain. J. Controlled Release 2015, 30-40.

(23) Needham, D.; Anyarambhatla, G.; Kong, G.; Dewhirst, M. W. A New Temperature-Sensitive Liposome for Use with Mild Hyperthermia: Characterization and Testing in a Human Tumor Xenograft Model. Cancer Res. 2000, 60, 1197-1201.

(24) McEwan, C.; Owen, J.; Stride, E.; Fowley, C.; Nesbitt, H.; Cochrane, D.; Coussios, C. C.; Borden, M.; Nomikou, N.; McHale, A. P.; Callan, J. F. Oxygen Carrying Microbubbles for Enhanced Sonodynamic Therapy of Hypoxic Tumours. J. Controlled Release 2015, 203, 51-56. 
(25) Lee, L.; Cavalieri, F.; Ashokkumar, M. Exploring New Applications of Lysozyme-Shelled Microbubbles. Langmuir 2019, 9997-10006.

(26) Oeffinger, B. E.; Vaidya, P.; Ayaz, I.; Shraim, R.; Eisenbrey, J. R.; Wheatley, M. A. Preserving the Integrity of Surfactant-Stabilized Microbubble Membranes for Localized Oxygen Delivery. Langmuir 2019, 10068-10078.

(27) Sennoga, C.; Mahue, V.; Loughran, J.; Casey, J.; Seddon, J. M.; Tang, M.; Eckersley, R. J. On Sizing and Counting of Microbubbles Using Optical Microscopy. Ultrasound Med. Biol. 2010, 36, 20932096.

(28) Hitchcock, K. E.; Caudell, D. N.; Sutton, J. T.; Klegerman, M. E.; Vela, D.; Pyne-Geithman, G. J.; Abruzzo, T.; Cyr, P. E. P.; Geng, Y. J.; McPherson, D. D.; Holland, C. K. Ultrasound-Enhanced Delivery of Targeted Echogenic Liposomes in a Novel Ex Vivo Mouse Aorta Model. J. Controlled Release 2010, 144, 288-295.

(29) Härmark, J.; Hebert, H.; Koeck, P. J. B. Shell Thickness Determination of Polymer-Shelled Microbubbles Using Transmission Electron Microscopy. Micron 2016, 85, 39-43.

(30) Laskowski, L.; Burk, R. E. The Probable Length of Hydrocarbon Chains. J. Chem. Phys. 1939, 7, 465-469.

(31) Borden, M. A.; Longo, M. L. Dissolution Behavior of Lipid Monolayer-Coated, Air-Filled Microbubbles: Effect of Lipid Hydrophobic Chain Length. Langmuir 2002, 18, 9225-9233.

(32) Feshitan, J. A.; Chen, C. C.; Kwan, J. J.; Borden, M. A. Microbubble Size Isolation by Differential Centrifugation. J. Colloid Interface Sci. 2009, 329, 316-324.

(33) Sezgin, E.; Waithe, D.; Bernardino De La Serna, J.; Eggeling, C. Spectral Imaging to Measure Heterogeneity in Membrane Lipid Packing. ChemPhysChem 2015, 16, 1387-1394.

(34) Parasassi, T.; De Stasio, G.; Ravagnan, G.; Rusch, R. M.; Gratton, E. Quantitation of Lipid Phases in Phospholipid Vesicles by the Generalized Polarization of Laurdan Fluorescence. Biophys. J. 1991, 60, 179-189.

(35) Aron, M.; Browning, R.; Carugo, D.; Sezgin, E.; Bernardino de la Serna, J.; Eggeling, C.; Stride, E. Spectral Imaging Toolbox: Segmentation, Hyperstack Reconstruction, and Batch Processing of Spectral Images for the Determination of Cell and Model Membrane Lipid Order. BMC Bioinf. 2017, 18, 254.

(36) Carugo, D.; Owen, J.; Crake, C.; Lee, J. Y.; Stride, E. Biologically and Acoustically Compatible Chamber for Studying Ultrasound-Mediated Delivery of Therapeutic Compounds. Ultrasound Med. Biol. 2015, 41, 1927-1937.

(37) Riccardi, C.; Nicoletti, I. Analysis of Apoptosis by Propidium Iodide Staining and Flow Cytometry. Nat. Protoc. 2006, 11, 14581461.

(38) Uggeri, J.; Gatti, R.; Belletti, S.; Scandroglio, R.; Corradini, R.; Rotoli, B. M.; Orlandini, G. Calcein-AM Is a Detector of Intracellular Oxidative Activity. Histochem. Cell Biol. 2000, 122, 499-505.

(39) Bittman, R.; Blau, L. Phospholipid-Cholesterol Interaction. Kinetics of Water Permeability in Liposomes. Biochemistry 1972, 11, 4831-4839.

(40) Chapman, D. Phase Transitions and Fluidity Characteristics of Lipids and Cell Membranes. Q. Rev. Biophys. 1975, 8, 185.

(41) Stoll, C.; Stadnick, H.; Kollas, O.; Holovati, J. L.; Glasmacher, B.; Acker, J. P.; Wolkers, W. F. Liposomes Alter Thermal Phase Behavior and Composition of Red Blood Cell Membranes. Biochim. Biophys. Acta, Biomembr. 2011, 1808, 474-481.

(42) Dwight, J. F. S. J.; Hendry, B. M. Effects of Membrane Incorporation of Short-Chain Phospholipids on Sodium Pump Function in Human Erythrocytes. Clin. Chim. Acta 1995, 243, 73-85.

(43) Ferrell, J. E.; Lee, K. J.; Huestis, W. H. Lipid Transfer between Phosphatidylcholine Vesicles and Human Erythrocytes: Exponential Decrease in Rate with Increasing Acyl Chain Length. Biochemistry 1985, 24, 2857-2864.

(44) Lyon, P. C.; Griffiths, L. F.; Lee, J.; Chung, D.; Carlisle, R.; Wu, F.; Middleton, M. R.; Gleeson, F. V.; Coussios, C. C. Clinical Trial Protocol for TARDOX: A Phase I Study to Investigate the Feasibility of Targeted Release of Lyso-Thermosensitive Liposomal Doxorubicin
(ThermoDox) Using Focused Ultrasound in Patients with Liver Tumours. J. Ther. Ultrasound 2017, 5, No. 28.

(45) Ríos-Marco, P.; Marco, C.; Gálvez, X.; Jiménez-López, J. M.; Carrasco, M. P. Alkylphospholipids: An Update on Molecular Mechanisms and Clinical Relevance. Biochim. Biophys. Acta, Biomembr. 2017, 1859, 1657-1667.

(46) Jaffrès, P.-A.; Gajate, C.; Bouchet, A. M.; Couthon-Gourvès, H.; Chantôme, A.; Potier-Cartereau, M.; Besson, P.; Bougnoux, P.; Mollinedo, F.; Vandier, C. Alkyl Ether Lipids, Ion Channels and Lipid Raft Reorganization in Cancer Therapy. Pharmacol. Ther. 2016, 165, 114-131.

(47) Nakata, M.; Tanimura, N.; Koyama, D.; Krafft, M. P. Adsorption and Desorption of a Phospholipid from Single Microbubbles under Pulsed Ultrasound Irradiation for UltrasoundTriggered Drug Delivery. Langmuir 2019, 10007-10013.

(48) Farook, U.; Stride, E.; Edirisinghe, M. J. Preparation of Suspensions of Phospholipid-Coated Microbubbles by Coaxial Electrohydrodynamic Atomization. J. R. Soc., Interface 2009, 6, 271-277.

(49) George, S. D.; Chidangil, S.; Mathur, D. Minireview: LaserInduced Formation of Microbubbles - Biomedical Implications. Langmuir 2018, 10139-10150.

(50) Mahalingam, S.; Xu, Z.; Edirisinghe, M. Antibacterial Activity and Biosensing of PVA-Lysozyme Microbubbles Formed by Pressurized Gyration. Langmuir 2015, 31, 9771-9780.

(51) Chen, Z.; Pulsipher, K. W.; Chattaraj, R.; Hammer, D. A.; Sehgal, C. M.; Lee, D. Engineering the Echogenic Properties of Microfluidic Microbubbles Using Mixtures of Recombinant Protein and Amphiphilic Copolymers. Langmuir 2019, 10079-10086.

(52) Parhizkar, M.; Stride, E.; Edirisinghe, M. Preparation of Monodisperse Microbubbles Using an Integrated Embedded Capillary T-Junction with Electrohydrodynamic Focusing. Lab Chip 2014, 14, 2437-2446.

(53) Unnikrishnan, S.; Du, Z.; Diakova, G. B.; Klibanov, A. L. Formation of Microbubbles for Targeted Ultrasound Contrast Imaging: Practical Translation Considerations. Langmuir 2018, 10034-10041. 